\title{
Conservative Approach of a Severe Case of Osteoradionecrosis of the Mandible- Case Report
}

\author{
Touil Dorsaf1, Hassouna Mariam El Ons², Abdel Mouleh Yosri'3, Oualha Lamia ${ }^{4}$ \\ ${ }^{1}$ Assistant Professor, Department of Oral Medicine and Oral Surgery, University Hospital Sahloul Sousse, \\ Tunisia. \\ 2,3Resident, Department of Oral Medicine and Oral Surgery, University Hospital Sahloul Sousse, Tunisia. \\ ${ }^{4}$ Professor, Department of oral medicine and oral surgery, University Hospital Sahloul Sousse, Tunisia.
}

Case Report

Address for Correspondence Author

Dr. Touil Dorsaf; Department of Oral Medicine and Oral Surgery, University Hospital Sahloul Sousse, Tunisia.

E-mail: touil.oueslati.dorsaf@gmail.com

Crossref doi: https://doi.org/10.36437/ijdrd.2020.2.3.G

\begin{abstract}
Osteoradionecrosis (ORN) of the jaw is a severe, complex, and multifactorial complication of radiotherapy (RT) for head and neck cancers. Most cases of ORN develop during the first 3 years with a peak between 6 months and 2 years after completion of RT however the patient is considered at risk of developing this condition many years later. The management of ORN is usually based on conventional medical care by focusing on comorbidity factors, However, in some refractory ORN cases, the condition requires extensive surgical resection. Based on the "the radiation-induced fibro-atrophic theory" introduced by Delanian, a new conservative approach of ORN seems to be promising by combining antioxidant agents: pentoxifylline and Tocopherol (PtxE) in the treatment of severe cases of ORN.

The aim of this paper was to report the case of a severe case of ORN of the mandible stabilized by a conservative approach combining antibiotics, antiseptic agent as well as antioxidant therapy (PtxE), and to discuss the efficacy of this approach.
\end{abstract}

Keywords: Osteoradionecrosis (ORN), fibro-atrophic theory, Mandible, Conservative Approach.

\section{Introduction}

Osteoradionecrosis (ORN) of the jaw is a severe, complex, and multifactorial complication of radiotherapy (RT) for head and neck cancers. According to the most recent literature, ORN of the jaws was defined as "an exposed irradiated bone that fails to heal over a period of 3 months without any evidence of persisting or recurrent tumor". ${ }^{1}$

Ewing ${ }^{2}$ was the first to use the term 'radiation osteitis' to describe changes that occur in the bone after the radiotherapy. Later, several terms were used to name these changes in the irradiated bone, such as radiation osteitis, ORN, and avascular bone necrosis. ${ }^{3}$ 
In 1983, Marx defined ORN as 'an area larger than $1 \mathrm{~cm}$ of exposed bone in a field of irradiation that failed to show any evidence of healing for at least 6 months'.4

In 1987, Marx and Johnson suggested the definition of ORN as: "The exposure of non-viable bone which fails to heal without intervention'. ${ }^{5}$

Most cases of ORN develop during the first 3 years (70\%-94\%), with a peak between 6 months and 2 years after completion of RT, however, the patient is considered at risk of developing this condition many years later. ${ }^{6}$

The most important inducing factor is a surgical trauma, commonly a dental extraction. However, Other trauma causing mechanical injuries as well as poor dental status can induce the necrosis as well (1). In less than $10 \%$ of the cases, ORN occurs spontaneously. ${ }^{7}$

The pathophysiology of ORN has evolved through the time. First, microorganisms were considered to play an important role in the occurrence of ORN in irradiated bone. Meyer concluded to his theory of a classic triad of radiation, trauma, and infection. ${ }^{8}$ Later, Marx proposed the $3 \mathrm{H}$ theory of hypoxia, hypocellularity, and hypovascularity. ${ }^{3}$

Recently, a new theory was proposed by Delanian9: "the radiation-induced fibroatrophic theory". This theory proposed that the progression of ORN is due to the activation and the deregulation of fibroelastic activity that leads to atrophic tissue in the irradiated area.

The management of ORN is usually based on conventional medical care focusing on comorbidity factors. Basically, by the optimization of oral hygiene, infection control with antibiotics and antiseptic agents, and the removal of the devitalized bone when possible. However, the Refractory ORN requires extensive surgical resection. ${ }^{10}$

Based on the recent theory of the radiation-induced fibroatrophy, a combination of an antioxidant treatment combining pentoxifylline and tocopherol (PtxE) with or without the adjunction of Clodronate ( PENTOCLO) was suggested and tested on phase II clinical trial.11

However, the reported results remained controversial. In some cases, there were good results with a healing rate that reached $100 \%{ }^{12}$ but in other reports, there was only little improvement with higher toxicity. ${ }^{13}$

The aim of this paper was to report the case of a severe ORN of the mandible stabilized by a conservative approach combining antibiotic, antiseptic as well as antioxidant (PtxE) therapy, and to discuss the efficiency of this approach compared to a more radical one.

Case report: A 59-year-old female patient was referred by her dentist for "severe pain due to a purulent lesion on the right side of the sub-mandibular area.

The medical history of the patient revealed that she was diagnosed with a T2B N1M0 undifferentiated carcinoma of nasopharyngeal type (UCNT) in 2012 and treated with combined Chemotherapy and conventional curative radiotherapy. The Total dose of the delivered radiotherapy was 74Gy with a fractioning of 2Gy per day, 5 days per week.

No systemic disease or chronic conditions were present. 
The extra-oral examination of the patient revealed a painful, purulent, and indured lesion of her right submandibular area. (figure1 and 2). No recent teeth pain or extraction was reported.

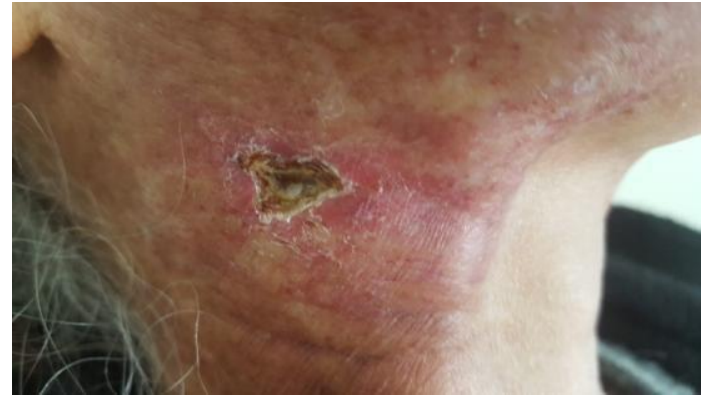

Figure1 and 2: Extra Oral Examination: Painful Purelent Lesion of the Sub- Mandibular area.

The patient had a severe limitation of her mouth opening as well as severe xerostomia that were both attributed to the side effects of the radiotherapy. (figure4)

The intra-oral examination, which was difficult to perform given the severe trismus, showed: very poor oral hygiene. All the remaining teeth were severely decayed. (figure 2 and 3 ).

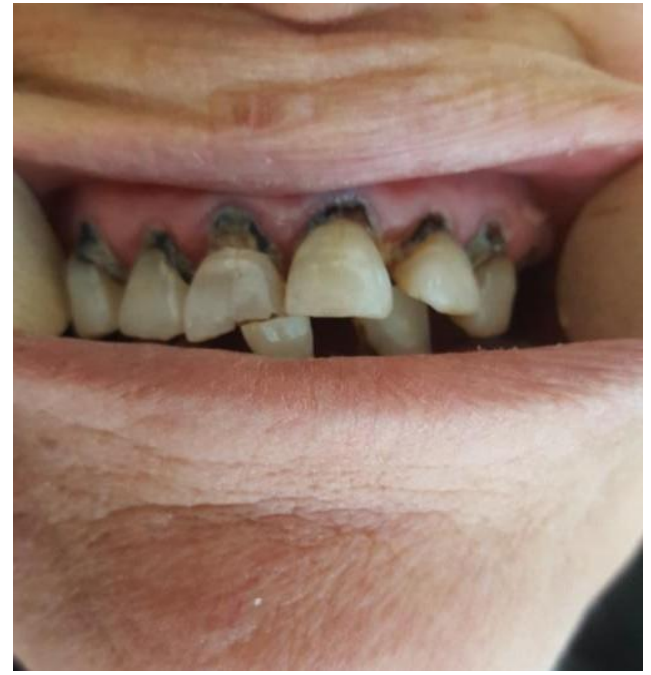




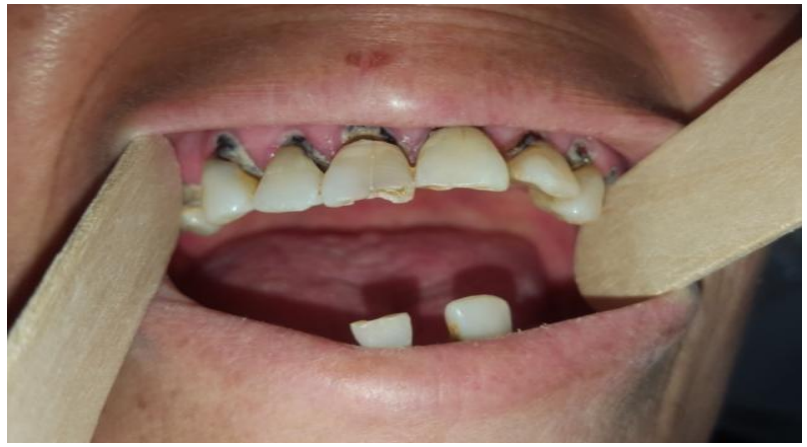

Figure 3 and 4: Intra Oral Examination: Decayed Teeth and Severe Xerostomia

The purulent exudate was evident in the socket of 47. A small area of exposed bone was also noticed in the lower vestibule.

The panoramic radiograph showed that the roots of 47.46 .45 and 44 were present and presented radiolucent images in their apical region combined with confluent radio opacities (figure5).

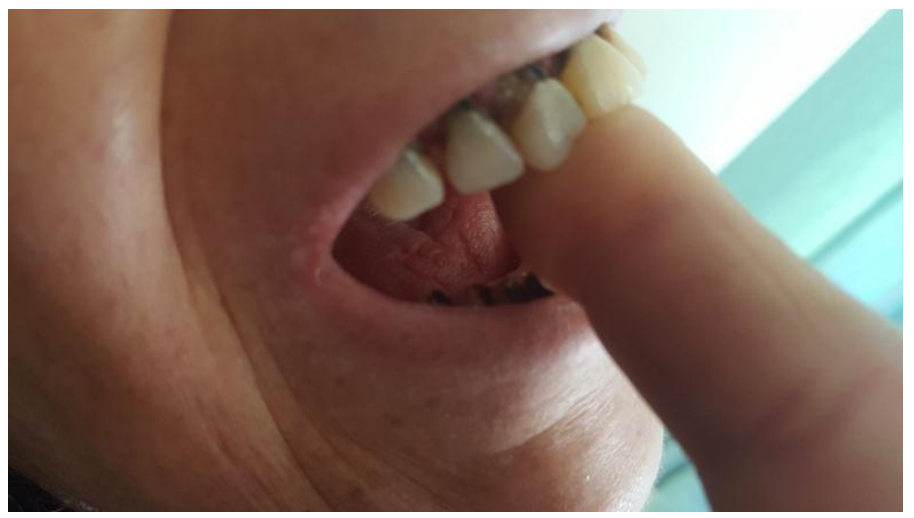

Figure5: Severe Limitation of the Mouth Opening

CT scan showed osseous abnormalities of the entire mandible, with focal lytic areas as well as cortical interruptions and loss of the spongiosa trabeculation on the symptomatic side. (figure6) The diagnosis of stage III of Epstein ORN of the mandible with submandibular fistulae was made. Our therapeutic approach consisted in the prescription of Amoxicillin-Clavulanic Acid (Augmentin) 3g/day in association with metronidazol (Flagyl) $1.5 \mathrm{~g} /$ day for 2 weeks.

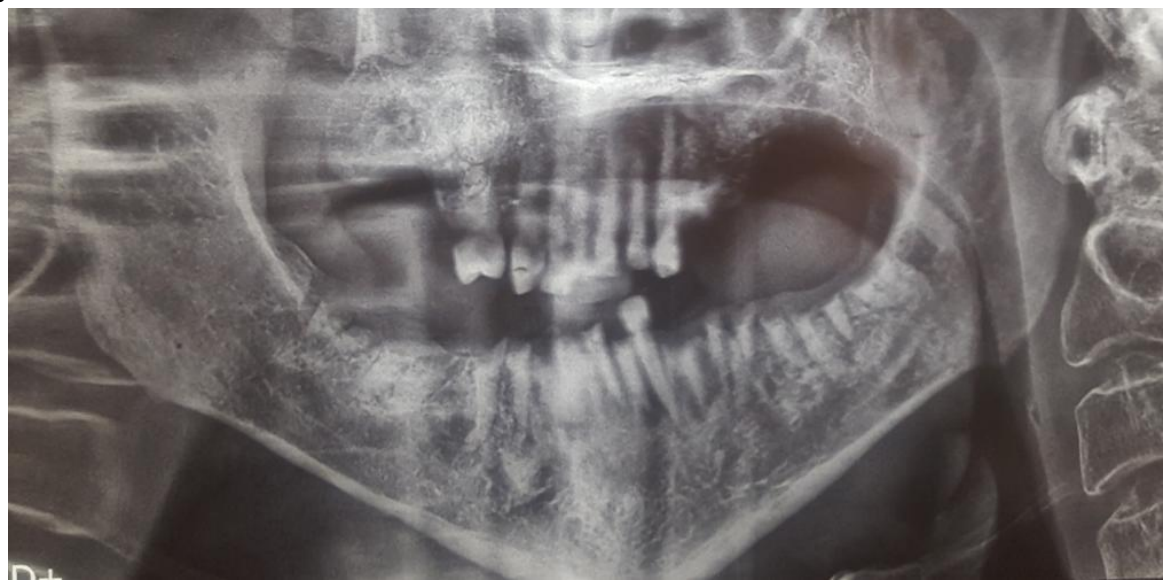

Figure 6: Panoramic Radiograph Showing Increased Radiodensity and Mixed Radioopaque/Radiolucent Lesion 
We also prescribed Pentoxifylline (Torental) $800 \mathrm{mg} /$ day, Tocopherol 1000UI/day for 2 weeks Paracetamol 3g /day and Chlorhexidine mouthwashes 4 times/day. Pilocarpine 4mg 5 times daily The extraction of 47 was postponed.

Ten days later, we noticed an improvement in the fistulae which showed signs of resolution of the infection (figure 7 and 8). The patient also reported a decrease in her pain and an improvement of her mouth opening (figure 9).

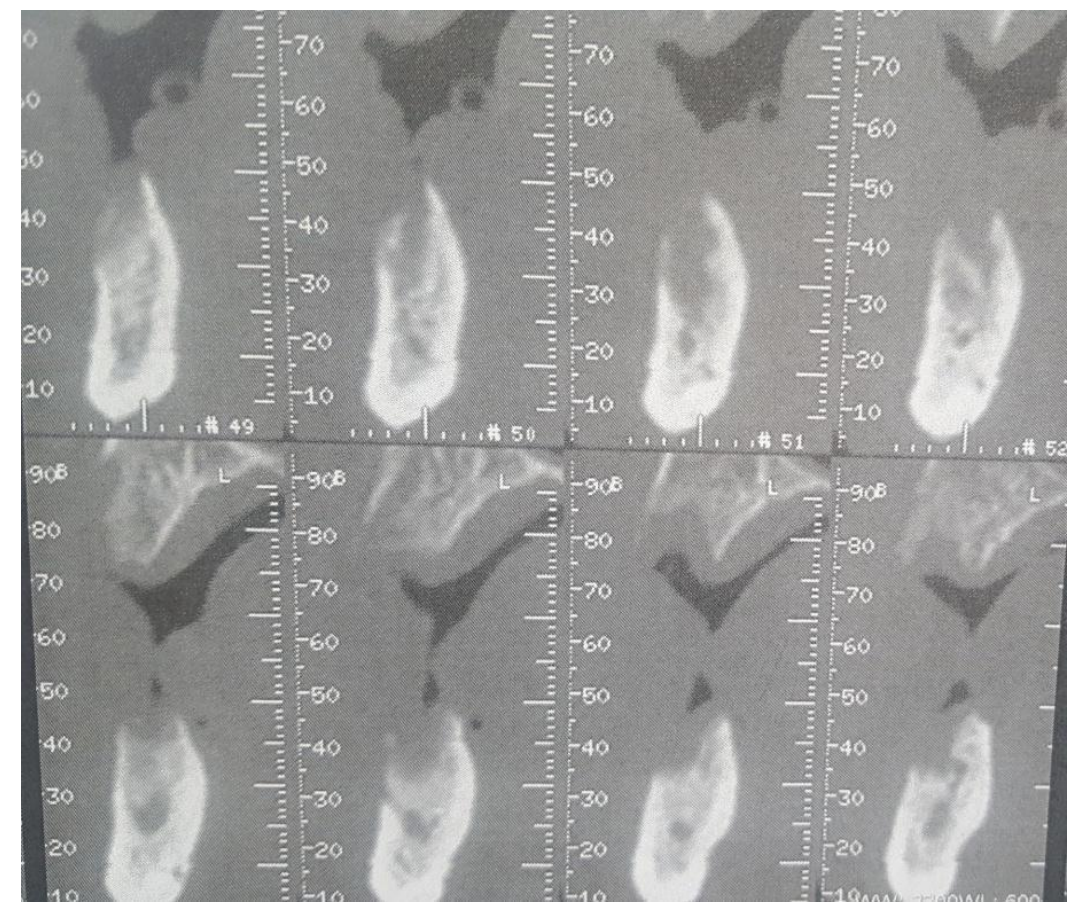

Figure7: CT Scan Cortical Bone Destruction

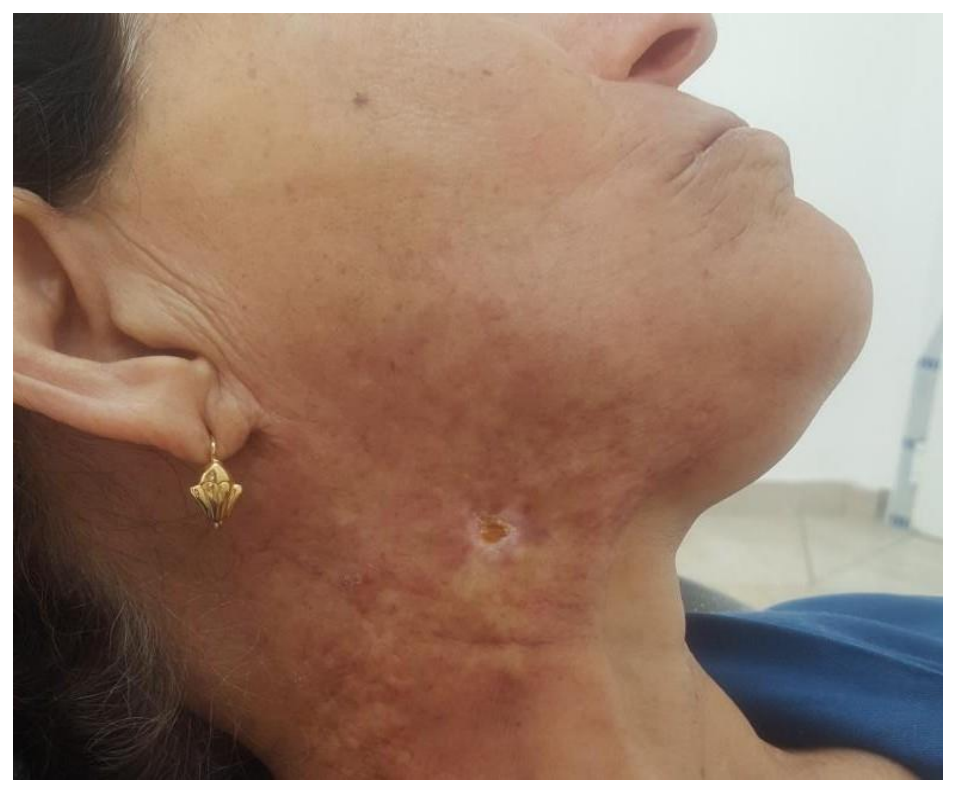




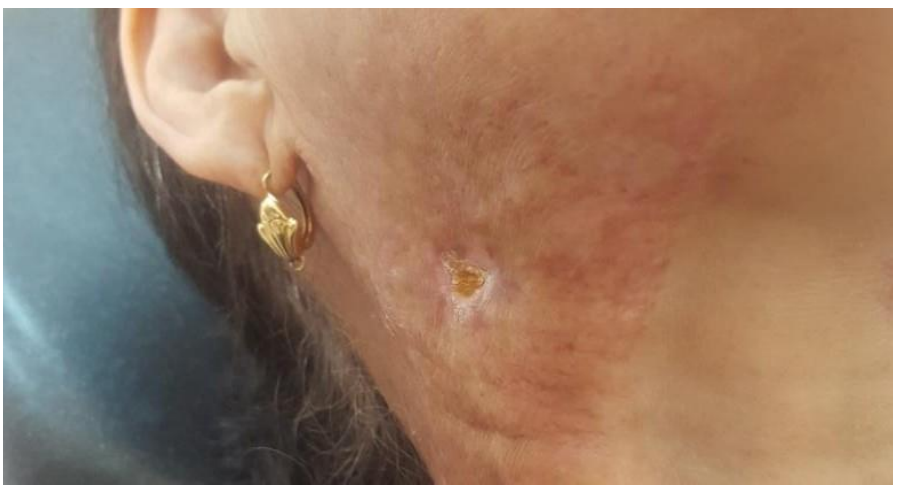

Figure 8 and 9: Control Images Showing Improvement of the Condition with Regression of the Infection and the Inflammation.

However, on the intraoral examination, we noticed that the socket of the tooth 47 was still infected. So we decided to perform the extraction of 47 under local anesthesia followed by a meticulous curettage of the socket using an antiseptic solution. No modification of medical therapy was made.

The patient was received 3 weeks later. She reported a significant improvement in her pain and her fistulae. The socket of 47 showed signs of good cicatrisation. However, the patient reported a severe intolerance to Pentoxifylline in the form of severe nausea which led her to stop taking it.

The patient was asked to continue her antibiotherapy, Vit E, and the pilocarpine as well as the use of the mouth wash. However, the use of Ptx was interrupted. Follow-ups were programmed monthly.

Discussion: ORN of the jaws remains among the most commonly encountered and severe complications of radiotherapy for head and neck cancers. ${ }^{3}$

This condition occurs more frequently in the mandible because of its relatively poor blood supply, which is exclusively provided by the inferior alveolar artery..$^{15}$ Moreover, ORN is generally caused by local trauma such as a dental extraction however some other incriminated patient dependent risk factors are reported such as age, diabetes, collagen vascular disease but also poor oral hygiene and alcohol and tobacco consumption. ${ }^{15}$ In our case, no dental extraction was reported and the complication was attributed to bad oral hygiene.

Different staging and scoring systems of ORN have been proposed.

These systems are based either on the response to hyperbaric oxygen (HBO) therapy; Marx staging system (5) or on the degree of bone damage, clinical- radiological findings, duration of bone exposure and treatment required. ${ }^{16,17,18}$

In our case, the patient was considered as a stage III of Epstein (table I), since her condition was progressive with purulent exudates in both the fistulae and the socket of 47 as well as the severe pain and trismus.

In fact, the clinical symptoms of ORN vary but generally include ulceration of the mucosa with exposure of necrotic bone that fails to heal for more than 3 months as well as pain, trismus and suppuration which were the main symptoms of our patient. ${ }^{18}$ 


\begin{tabular}{|c|c|c|}
\hline Stage & Description & Symptoms \\
\hline$\underline{I}$ & Resolved healed & None \\
\hline$\underline{\mathbf{I}} \mathbf{a}$ & No pathologic fracture & None or Controlled \\
\hline$\underline{\text { Ib }}$ & Pathologic fracture & \\
\hline$\underline{\text { II }}$ & Chronic persistent non progressive & \\
\hline$\underline{\underline{\text { Ia }}}$ & No pathologic fracture & \\
\hline$\underline{\underline{I I b}}$ & pathologic fracture & Jaw Dysfunction \\
\hline$\underline{\underline{\mathrm{III}}}$ & Active progressive & Progressive \\
\hline$\underline{\text { IIIa }}$ & No pathologic fracture & \\
\hline$\underline{\underline{I I I b}}$ & Pathologic fracture & Jaw Dysfunction \\
\hline
\end{tabular}

Table I ORN staging according to Epstein ${ }^{17}$

The progression of ORN may lead to pathological fractures and local or systemic infection. Difficulties in mouth opening, mastication and speech frequently arise and affect severely the patient's quality of life. ${ }^{18}$

The diagnosis of septic ORN appears to be easier. Marked by pain as a primary symptom 17 However, in some cases, this complication is only revealed by radiographic images that show localized osteolytic areas, extensive osteolytic areas, sequestra and fracture. ${ }^{18}$

In our case, the radiographic alterations were in the form of increased radiodensity, as well as a mixed radioopaque/radiolucent lesion in which radiolucent areas represented bone destruction. (Figure 8)

The management of ORN is usually based on conventional medical care focusing on comorbidity factors. Basically by infection control with antibiotics and antiseptic agents as well as by the removal of the devitalized bone when possible. ${ }^{19}$

In our case, the infection was controlled with association of Amoxicillin - Clavulanic acid and Metronidazol as well as Antiseptic agent; Chlorohexidine mouth wash.

We also prescribed Pentoxifylline and Tocopherol (Vit E) following the protocol proposed by Delanian. ${ }^{11}$

Pentoxifylline (PTX), is an antioxidant agent generally used to treat vascular diseases such as intermittent claudication. In vivo, it has been reported to have an anti-TNFa effect, increase erythrocyte flexibility, vasodilate, and inhibit inflammatory reactions. ${ }^{11}$

The recommended dose used by the authors are: Pentoxifylline: 800mg /day, Tocopherol; 1000UI/day with a treatment duration that varies from 3 to 24 months. ${ }^{11,12}$

McLeod et al. reported in their study of 12 patients who had received this protocol for an average of 14.8 months that 5 of these patients improved, 5 remained stable, and 2 deteriorated, requiring microvascular reconstructive surgery. ${ }^{20}$

A modified protocol proposed by D'souza involved administering $800 \mathrm{mg} /$ day of pentoxifylline, $1000 \mathrm{IU} /$ day of tocopherol, and $100 \mathrm{mg} /$ day of doxycycline. With the use of this protocol, the cure rate reached $70 \%$ among patients with stage I and stage II disease, whereas the disease remained stable in $40 \%$ of patients in stage III. ${ }^{21}$

51 International Journal of Drug Research and Dental Science 
In another retrospective analysis of 62 patients with established ORN, led by Patel et al, the success rate reached 56\% in patients how received Ptx and Vit E association. ${ }^{22}$

Even if this protocol seemed to be safe with minimum side effects, ${ }^{23}$ our patients had developed a severe intolerance to pentoxifylline which justified the interruption of the treatment a few weeks later. Some other side effects of Ptx were reported in the literature such as vertigo, nausea and headache. ${ }^{24}$

In some cases of ORN, surgical approaches such as small sequesters removal and debridement of superficial bone until bleeding were also reported in many studies as conservative management. In advanced or refractory cases of ORN, surgical treatment, including microvascular reconstructive techniques for bone and soft tissue, remains the only option available. ${ }^{25}$

In our case, the extraction of the residual roots of 47 was indicated since they were infected with signs of alveolar abscess which also was incriminated in the onset of the ORN. however, no other surgical treatment was proposed due to the good results obtained with this conservative approach.

\section{Conclusion}

This case report showed that conservative treatment of ORN should be indicated and implemented whenever it is possible based on the infection control with antibiotic and antiseptics agent with minimal surgical debridement. This approach can improve the overall situation of the patient especially when the surgical treatment may cause severe morbidity and does not always guaranty the healing or the improvement of the condition.

\section{References}

1. Jacobson AS, Buchdinder D.. Paradigm shifts in the management of ORN of the mandible .oral oncol, 2010. https://doi.org/10.1016/j.oraloncology.2010.08.007

2. Titterington WP. Osteomyelitis and osteoradionecrosis of the jaws. J Oral Med1971 26:7-16. https://www.osti.gov/biblio/4770154-osteomyelitis-osteoradionecrosis-jaws

3. Koteswara Rao Nadella - Rama Mohan Kodali; Osteoradionecrosis of the Jaws: ClinicoTherapeutic Management: A Literature Review and Update Radiother Oncol. 2004 Nov; 73(2):119-31.

4. Marx RE; osteoradionecrosis : a new concept of its pathophysiology ; J Oral maxillofac Surg 1983; 41:283-8. https://doi.org/10.1016/0278-2391(83)90294-x

5. Marx RE, Johnson RP, Studies in the radiobiology of osteoradionecrosis and their clinical significance. https://doi.org/10.1016/0030-4220(87)90136-8

6. Costa DA, costa TP, Netto EC et al. new perspectives on the conservative management of osteoradionecrosis of the mandible : a literature review. Head Neck 2016; 38 (11):1708-16. https://doi.org/10.1002/hed.24495

7. Curi MM, Dib LL. Osteonecrosis of the jaws: a retrospective study of the background factors and treatment in 104cases.J oral maxillofac 1993; 94:140-7.

8. Meyer I. Infectious diseases of the jaws. J oral Surg 1970; 28(1):17-26. https://pubmed.ncbi.nlm.nih.gov/5262227/

9. Delanian S, Lefaix JL. The radiation induced fibroatrophic process: therapeutic perspective via the antioxidant pathway. Radiother Oncol 2004;73(2):119-31. https://doi.org/10.1016/j.radonc.2004.08.021 
10. Lyons A, Osher J, Warner E, Kumar R, Brennan PA, Osteoradionecrosis -a review of current concepts in defining the extent of the disease and a new classification proposal. Br J Oral Maxillofac Surg 2014 ; 52(5) : 392-5. https://doi.org/10.1016/j.bjoms.2014.02.017

11. Delanian S1, Depondt J, Lefaix JL.Major healing of refractory mandible osteoradionecrosis after treatment combining pentoxifylline and tocopherol: a phase II trial. Head Neck 2005; 27:114-23. https://doi.org/10.1002/hed.20121

12. Delanian S, Lefaix JL. Complete healing of severe osteoradionecrosis with treatment combinig pentoxifylline , tocopherol and clodronate. BrJ Radiol 2002;75; 467-9. https://doi.org/10.1259/bjr.75.893.750467

13. Dion MW, Hussey DH, Doornbos JF, V igliotti AP, Wen BC, Anderson B. Preliminary results of a pilot study of pentoxifylline in the treatment of late radiation soft tissues necrosis. Int J radiat 19(2)-401-7. https://doi.org/10.1016/0360-3016(90)90549-y

14. Bras J, de Jonge HK, Van Merkesteyn JP. osteonecrosis of the mandible: pathogenesis. Am J otolaryngol 1990; 11:244-50.https://doi.org/10.1016/0196-0709(90)90084-9

15. Beumer J, Harrison R, Sanders B, Kurrasch M.osteoradionecrosis: predisposing factors and outcomes therapy. Head Neck Surg 1984; 6: 819-27. https://doi.org/10.1002/hed.2890060404

16. Notani K, Yamazaki Y, Kitada $\mathrm{H}$ et al . Management of mandibular osteoradionecrosis corresponding to the severity of the osteonecrosis and the method of radiotherapy. Head Neck 2003 ;25(3):181-6. https://doi.org/10.1002/hed.10171

17. Epstein J, Wong F, Stevenson -Moore P. Osteonecrosis: Clinical experience and a proposal for classification. B j Oral Maxillo fac Surg 1987; 45:104-10. https://doi.org/10.1016/02782391(87)90399-5

18. Lyons A, Oser J, Warner E, Kumar R. Osteoradionecrosis -a review of current concepts in defining the extent of the disease and a new classification proposal $\mathrm{Br} \mathrm{J}$ oral maxillofac Surg2014;52(5):392-5. https://doi.org/10.1016/j.bjoms.2014.02.017

19. Kolokythas A, Rasmussen JT, Readon J, Feng C, management of osteoradionecrosid of the jasws with pentoxifylline-tocopherol: a systematic review of the literature and meta-analysis. J oral Maxillofac Surg 2018. https://doi.org/10.1016/j.ijom.2018.08.007

20. Mcleod NM, Pratt CA, Mellor TK, Brennan PA. Pentoxifylline and tocopherol in the management of patients with osteoradionecrosis. The Portsmouth experience. Br J oral Maxillofac Sur 2012; 50(1):41-4. https://doi.org/10.1016/j.bjoms.2010.11.017

21. D'Souza J, Hussey DH, Osborne JW. Changing trends and the management on the outcome of patients treated forosteoradionecrosis of the mandible; experience from a regional head and neck unit. Br J oral Maxillo fac Surg 2014; 52(4):356-62. https://doi.org/10.1016/j.bjoms.2014.01.003

22. Patel V, Gadiwalla Y, SAsson I. Use of pentoxifylline and tocopherol in the management of

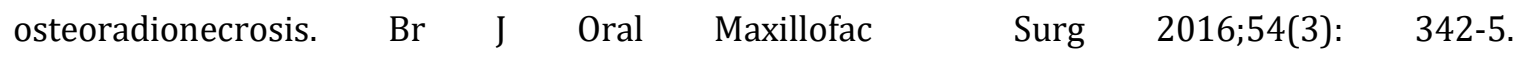
https://doi.org/10.1016/j.bjoms.2015.11.027

23. Patel V, Gadiwalla Y, SAsson I. Prophylactic use of pentoxifylline and tocopherol in patients who require dental extractions after radiotherapy for cancer of the head and neck. Br J Oral Maxillofac Surg2016; 54(5): 547-50. https://doi.org/10.1016/j.bjoms.2016.02.024

24. Kulkarni R, Gymermen, Gilbert k. The role of pentoxifylline- Tocopherol -Clodronate in osteoradionecrosis of the mandible. $\mathrm{Br} J$ Mxillofac Surg 2015; 53(10)62-3. https://doi.org/10.1016/i.bjoms.2015.08.078 
25. Reibeiro GH, Chrun ES, Dutra KL, Daniel FI. Osteonecrosis of the jaw; a review and update in etiology and treatment Braz J otorhinolaryngol 2018; 84(1):102-8. https://doi.org/10.1016/j.bjorl.2017.05.008

How to cite this Article: Touil Dorsaf1, Hassouna Mariam El Ons², Abdel Mouleh Yosri³, Oualha Lamia4: Conservative Approach of a Severe Case of Osteoradionecrosis of the Mandible-Case Report Int. J. Drug Res. Dental Sci., 2020; 2(3): 45-54.

Crossref doi: $h$ ttps://doi.org/10.36437/ijdrd.2020.2.3.G

Source of Support: Nil, Conflict of Interest: Nil.

Received: 16-7-2020 Revised: 19-8-2020 Accepted: 20-8-2020 